\title{
Kangaroo Mother Care - An Inspiration from Nature
}

\author{
Bindu Athoor,Shanfiyath C.V, Soumya Jose,P.M Kutty
}

\begin{abstract}
:
Aim: To study the effect of Kangaroo mother care (KMC) in comparison with conventional care $(C M C)$ at neonatal unit on feasibility, growth and hospital stay in very low birth weight infants. Methods: This was a pilot study conducted at a tertiary care neonatal unit. Low birth weight babies (weighing $<2000$ grams) were given kangaroo mother care $(K M C)$ for minimum of four hours. Feasibility of Kangaroo mother care,rate of weight gain, and duration of hospital stay were assessed.Results: .Total of 96 babies were there for the study, 41 in the $K M C$ group and 55 in the conventional method of care $(C M C)$. No parents opted out after starting KMC. $80.5 \%$ parents were able to give KMC for more than four hours. Weight of $63.4 \%$ babies were on or above the line in KMC group at discharge in contrast to 40\% babies in CMC. Mean length of hospital stay was 6.75 days in case of KMC group in contrast to 11.14 in CMC group.p value 0.09. CONCLUSION : Kangaroo mother care $(K M C)$ in the care of preterm babies is feasible in any setting after proper counselling of staff and parents.KMC is beneficial to the baby and the family as it improves weight gain and reduces length of hospital stay.
\end{abstract}

Keywords: kangaroo mother care, low birthweight,feasibility, hospital stay.

\section{Introduction}

Preterm birth is the largest direct cause of neonatal mortality, accounting for a estimated $27 \%$ of the 4 million neonatal deaths every year. Kangaroo mother care (KMC) is a type of care for preterm and premature infants whereby the infant is placed in an upright position against the parent's chest, with early skin-to-skin contact between the parent and infant. Mothers who practise KMC exhibit less maternal stress and fewer symptoms of depression, and have a better sense of the parenting role and more confidence in meeting their babies' needs than those who don't. Despite the apparent feasibility of KMC, currently, only a few preterm babies in low-income countries have access to this intervention. Knowledge of the effectiveness and safety of $\mathrm{KMC}$ in the community and home setting, and its effects on growth, is still incomplete ${ }^{1}$. Despite the said advantages of KMC, it is still not a widely practiced method of care for all the LBW infants in India ${ }^{(2)}$. Kangaroo mother care (KMC) is humane, low cost technique for care of preterm low birth weight infants which can be started early and can be easily done both in hospital and even at home after the discharge of the baby ${ }^{(3,4)}$. It is a powerful, easy-to-use method to promote the health and well-being of infants born preterm and LBW7. During KMC, mothers function somewhat like human incubators, providing physiological homeostasis, appropriate stimulation, and the main source of nutrition. Basic physiological variables such as temperature, oxygenation and heart rate are maintained within clinically acceptable limit in the kangaroo position ${ }^{(5)}$.

Design and study population: This was a prospective analytical study conducted for a period of one year from November 2012 to november 2013 at referral NICU of a multi-specialty teaching hospital in South India: Kangaroo mother care group-baies admitted during $1^{\text {st }}$ june2013 to $30^{\text {th }}$ November 2013 (6 months): conventional care group: $1^{\text {st }}$ november 2012 to $30^{\text {th }}$ april 2012 ( 6 months). $1^{\text {st }}$ may to $31^{\text {st }}$ may 2013 used for introducing the concept of KMC to the staff of icu and postnatal ward.

Inclusion and Exclusion criteria: Inclusion criteria were LBW babies with birth weight $<2000 \mathrm{gm}$ admitted in new born Nursery. Exclusion criteria were critically ill babies requiring ventillatory or ionotropic support or radiant warmer, babies with chromosomal and

life threatening congenital anomalies, babies whose mothers are critically ill and babies whose mothers do not consent for study.The present study was conducted in a setting where KMC was not practiced.During a one month period nursing staff and residents were received training on KMC, its use ,benefits and protocol was made.Once the babies are stable mothers were advised to start KMC. Before starting Kangaroo Mother Care, the method of care and

its benefi ts were explained to participating mothers and at least one other family member. Mothers and staff $\mathrm{s}$ were also informed and asked to look for dangers signs such as apnea, cyanosis during KMC so that action could be taken immediately. Once both baby and mother were ready, the KMC group was subjected to Kangaroo mother care of at least 6 hours per day in not more than 4 sittings, each sitting of at least 1 hour. During Kangaroo care, mother wore a loose shirt and the baby was held upright between the breasts and the limbs were flexed and the head was turned to one side not much fl exed or extended. Babies wore only diaper and a cap during the period of KMC. The blouse covered the infant's trunk and extremiti es but not the head. The baby was further supported by a long clothes which was wrapped around mother's chest from outside her 
shirt. Then mother was seated in a comfortable chair. The mother was encouraged to hold her baby in this position for at least 1 hour. If the baby passed urine and/or stool during the procedure and she felt discomfort she was asked to change the diaper and continue $\mathrm{KMC}$. When babies were not in $\mathrm{KMC}$, they were adequately clothed and kept covered. The mothers were provided KMC chart to keep the records of duration of KMC. In control group babies were adequately clothed and covered .Control group babies were those babies received conventional method only were selected from records in the 6 months period prior to initiating KMC and met the same enrollment criteria.Primary outcome measured was feasibility of Safety of the babies was ensured by nurses assigned to the baby and doctors in the NICU. Pulse oximeter was available for each baby even in historical cohort which recorded continuously oxygen saturation and heart rate. Any acute event could be immediately attended to. Recording of temperature of baby in kangaroo group was done before, during and after $\mathrm{KMC}$ with a thermometer kept in axilla for 5 minutes. When not in $\mathrm{KMC}$, temperature was taken every 4 hours. Baseline characteristics of both groups were recorded in detail including name,gestational age bin weeks,birth weight,mode of delivery, APGAR score and mother's information (name, age, gravida/parity). All babies were monitored daily for any signs of infection/ sepsis, breast feeding, jaundice and weight gain. Daily weight was recorded using digital weighing machine with 5gram accuracy. Length (measured using infantometer) and occipitofrontal circumference measured weekly and plotted weekly on Wright's postnatal growth chart ${ }^{(6)} \mathrm{At}$ discharge weight, type of feeds, post menstrual age at discharge and stress of mother recorded. Stress was assessed by simple questionnaire of yes or no. Principal investigator recorded the outcomes.Primary outcome was the feasibility of KMC in a hospital in a rural area that is measured by $\mathrm{KMC}$ giving hours and number of family opting out from care giving.Secondary outcome were 1.duration of hospital stay(no.of days in hospital) 2.weight on discharge(drop from reference line on discharge in Wrights postnatal growth chart).

\section{Statistical Methods}

As this was a pilot study ,all babies fulfilled inclusion criteria during the study period were included in the study. Comparisons between study groups for discrete variables were performed with the chi-square or Fisher's exact test. Continuous variables were compared by Student's t-test or nonparametric tests, when appropriate.

\section{Results}

In this study, during the study period 130 neonates were there with birth weight below2000grams. 32 were excluded from the study . 2 babies were expired.Total of 96 babies were there for the study, 41 in the $\mathrm{KMC}$ group and 55 in the conventional method of care (CMC).

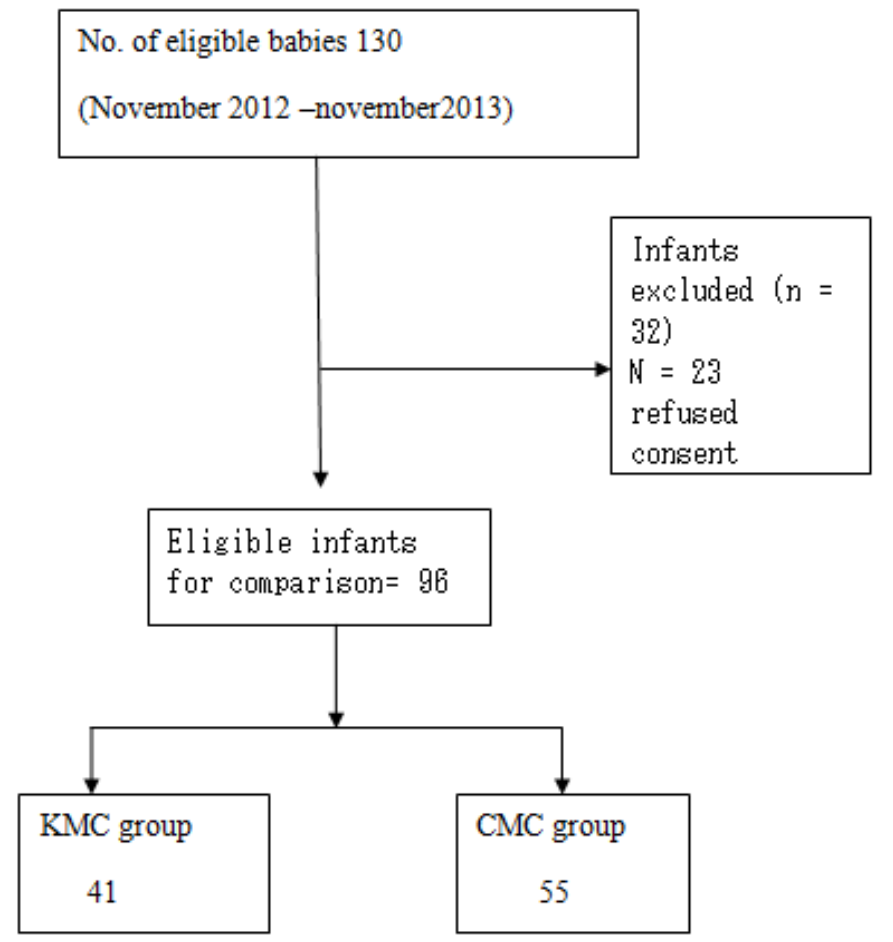

Table 1 displays baseline neonatal characteristics in two groups. All characteristics were comparable between 2 groups 
Table 1.Baseline characteristics

Variable

Birth weight $(\mathrm{g})($ mean $\pm \mathrm{SD})$

$\mathrm{KMC} \mathrm{n}=41(\mathrm{n}, \%)$

$1465+/-350$

$32.70+/-2.0$

$($ mean $\pm \mathrm{SD})$

Male sex

Intra uterine growth restriction

Weight at enrolment

Caesarean delivery

$21(51.2 \%)$

$7(17 \%)$

$1320+/-240$

$41.4 \%$

Active resuscitation at birth $\mathrm{n}(\%) \quad 6(14.6 \%)$

Education below 10th grade n (\%) 9(21.9)

Outcomes

$\begin{array}{cc}\mathrm{CMC} \mathrm{n}=55 \quad(\mathrm{n}, \%) & p \text { value } \\ 1620+/-225 & >0.05 \\ 33.10+/-1.8 & >0.05 \\ & \\ 30(56 \%) & >0.05 \\ 11(20 \%) & >0.05 \\ 1510+/-270 & >0.05 \\ 41,8 \% & >0.05 \\ 7(12.7 \%) & >0.05 \\ 13(23.6 \%) & >0.05\end{array}$

No parents opted out after starting KMC . 80.5\% parents were able to give KMC for more than four hours. Most babies were started KMC by a median age of 72 hours.

TABLE 2. feasibility ( CARE GIVING HOURS/ DAY )

\begin{tabular}{|c|c|}
\hline CARE GIVING (HOURS/ DAY) & KMC $(\mathrm{n}=41)$ \\
\hline$\geq 12$ & $5(12.2 \%)$ \\
\hline $4-12$ & $33(80.5 \%)$ \\
\hline$<4$ & $3(7.3 \%)$ \\
\hline
\end{tabular}

Weight on discharge

Weight of $63.4 \%$ babies were on or above the reference line in $\mathrm{KMC}$ group.

TABLE 16. WEIGHT AT DISCHARGE ON WRIGHTS POST NATAL GROWTH CHART

\begin{tabular}{|c|c|c|c|}
\hline $\begin{array}{c}\text { WEIGHT AT } \\
\text { DISCHARGE }\end{array}$ & $\begin{array}{c}\text { KMC } \\
(\mathrm{n}=41) \\
\text { No }(\%)\end{array}$ & $\begin{array}{c}\text { CMC }(\mathrm{n}=55) \\
\text { No }(\%)\end{array}$ & \\
\hline $\begin{array}{c}\text { ON OR ABOVE } \\
\text { RL }\end{array}$ & $26(63.4)$ & $22(40.0)$ & \\
\hline BELOW RL & $15(36.5)$ & $33(60.0)$ & \\
\hline TOTAL & 41 & 55 & $<0.05$ \\
\hline
\end{tabular}

RL:reference line in wrights postnatal growth chart

Duration of hospital stay

Mean length of hospital stay was 6.75 days in case of KMC group in contrast to 11.14 in CMC group.p value 0.09 . Breast feeding rates were analysed in prospective $\mathrm{KMC}$ cohort of babies.85.3\% were discharged exclusively on breast milk. Rest were on breast feeds and formula feeds,

\section{Discussion:}

Kangaroo mother care has been proposed as an alternative method for caring low birth weight neonate. The method was first implemented by Roy and Martinez in 1979 at Maternal and Child Institute of of Bogota, Colombia .It consists skin to skin contact, exclusive breast feeding and early discharge ${ }^{.7,8}$. Feasibility was assessed by care giving hours / day, parents opting out and stress expressed at time of discharge. $80.5 \%$ of parents could manage to spend $4-12$ hours /day, 12.2\% were able to spend $>12$ hours /day. One inhibitory factor was distance between nicu and maternal ward in our hospital. Second issue was, most parents were belongs to lower socioeconomic status in the rural area,they were in general ward and the privacy for mothers was an issue.Providing separate maternal ward near to nicu was considered after this study. So it is clear from our study that KMC is feasible in any setting. Various other studies had shown that KMC babies had better average weight gain per day. A study done by Suman Rao et al. from Mumbai India showed average weight gain of 23,99 grams in KMC groups. ' Similarly, experience of Gupta M. et al. from Rajasthan India,

showed average weight gain of 21.3 grams/day ${ }^{10}$ and weight gain was noticed in study by Ramanathan et al also.In our study weight of $63.6 \%$ were on or above the reference line in wright s postnatal growth chart. This reflects that $\mathrm{KMC}$ is beneficial for the babies weight gain.KMC might have increased breast feeding rates in 
the mother. Adequate warmth and breast feeding may be the contributing factors for weight gain. Gupta M, Jora $\mathrm{R}$ et al observed mean duration of hospital stay was 15.5 days. ${ }^{10}$. Average day of hospital stay was $27.2+-7$ days in study by Ramanathan et al ${ }^{11}$ Similarly, KMC in infants in Merida had discharged at 13.4 days after enrolment. ${ }^{12}$.In the present study duration of hospital stay was 11.14 days in conventional care group.in contrast to 6.75 days in KMC group and this was statistically significant. KMC reduced the number of total days in the hospital ,this would indirectly reduced cost of care and stress of parents. Sunil Ghavane also shown that kangaroo mother care in the Kangaroo ward is as effective as conventional care in the neonatal unit without any increase in morbidity or mortality in stable VLBW infants ${ }^{(13}$ ). LBW babies less than 2000 gm who receive KMC show better weight gain and have less incidence ofhypothermia than those who do not receive KMC ${ }^{(14) .}$ The results of our study were comparable with the above studies. Morbidities were sepsis for 2 babies apnoeas for 1 baby and cold stress for 4 babies after starting KMC. Then KMC was withheld till the problem has resolved.Strength of our study is, to our knowledge this is the first study conducted in a instituition in the rural area of southern india demonstrating the feasibility of KMC.We could also demonstrate better weight gain and less hospital stay for KMC babies.Limitation was as this was a pilot study we have not calculated the sample size. Randomised control study should be planned to observe more reliable effect on weight gain and morbidity.

\section{Conclusion}

Kangaroo mother care (KMC) in the care of preterm babies is feasible in any setting after proper counselling of staff and parents.KMC is beneficial to the baby and the family as it improves weight gain and reduces length of hospital stay.

\section{Acknowledgement}

Authors would like to express their gratitude to all the staffs of the baby unit and KMC ward and also to the mothers and their babies who participated in this study.

Abbreviations: Kangaroo mother care KMC. , Conventional medical care; $\mathrm{CMC}$

\section{References}

[1]. Shrivastava SR,et al. Utility of kangaroo mother care in preterm and low birthweight infants. S Afr Fam Pract 2013;55(4):340-344

[2]. Singh M. Temperature regulation. In: Singh M, editor. Care of the newborn, 5th ed. New Delhi: Sagar Publications, 2000 : $190-7$.

[3]. Catianeo A, Davanzo R, Uxa F and Tamburlini G. Recommendations for the implementation of Kangaroo Mother Care for low birth weight infants. Acta Paediatr, 1998;87:440-45.

[4]. World Health Organiza? on. Kangaroo mother care: a practical guide. Department of Reproductive Health and Research, WHO, Geneva.2003

[5]. Charpak N, Ruiz JG, Zupan J, Catianeo A, Figueroa Z, Tessier R et al Kangaroo Mother Care: 25 years After. Acta Paediatrica 2005;94:514-522.

[6]. Wright K, Dawson JP, Fallis D, Vogt E, Lorch V. New postnatal growth grids for very low birth weight infants. Pediatrics1993; 91:922-6

[7]. Whitelaw A, Sleath K. Myth of Marsupial Mother; Home Care of very Low Birth Weight Babies, in Bogota, Columbia. Lancet 1985; 25: 1206-1208.

[8]. Charpak N, Figueroa Z. Kangaroo Mother Care Programme Practical Rules. Bogota, Columbia: Kangaroo Foundation; 2001

[9]. Suman RP, Udani R, Nanavati R. Kangaroo Mother Care for Low Birth Weight Infants: A Randomized Controlled Trial. Indian Pediatr 2008;45(1): 17-23.

[10]. Gupta M, Jora R, Bhatia R. Kangaroo Mother Care (KMC) in LBW Infants- A Western Rajasthan Experience. Indian J Pediatr 2007; 74(8):747-9.

[11]. Ramanathan K, Paul VK, Deorari AK, Taneja U and George G. Kangaroo Mother Care in Very Low Birth Weight Infants. Indian J Pediatr 2001; 68(11):1019-1023.

[12]. Cattaneo A, Davanzo R, Worku B, Surjono A, Echeverria M, Bedri A, Haksari E,

[13]. Osorno L, Gude B, Setyowireni D, Quintero S, Tamburlin G. Kangaroo Mother Care for Low Birth Weight Infants: A Randomized Controlled Trial in Different Settings. Acta Paediatrica 1998; 87(9): 976-985.

[14]. Sunil Ghavane, Srinivas Murki. Kangaroo Mother Care in Kangaroo ward for improving the growth and breastfeeding outcomes when reaching term gestational age in very low

[15]. birth weight infants; Acta Pædiatrica 2012 101, pp. e545-e549

[16]. Acharya N1, Singh RR2, Bhatta NK3, Poudel PRandomized Control Trial of Kangaroo Mother Care in Low Birth Weight Babies at a Tertiary Level Hospital. J Nepal Paediatr Soc 2014;34(1):18-23. 